\title{
NIVEL DE ANSIEDAD Y PROCESO DE AFRONTAMIENTO EN ADULTOS MAYORES ATENDIDOS EN UN CENTRO DE SALUD ICA PERÚ 2017.
}

\author{
ANXIETY LEVEL AND COURTING PROCESS IN ELDERLY ADULTS SERVED AT ICA \\ PERU 2017 HEALTH CENTER.
}

Sauñe-Oscco Wilson E. ${ }^{\text {,b }}$, Martínez Paucar Félix ${ }^{1, a}$, Oscco-Torres Olinda ${ }^{1, b}$, Ramos-Cajo Astrid ${ }^{\text {,b }}$.

1. Docente de la Universidad Privada San Juan Bautista, Filial Ica.

a. Médico Cirujano

b. Licenciado en Enfermería del Hospital Santa María del Socorro de Ica, Perú.

c. Especialista en Cuidados gerontológicas

doi: https://doi.org/10.35563/rmp.v8i1.11

\section{Correspondencia:}

Jorge Ybaseta Medina.

Domicilio: Urb. Villa del Médico B-7, Ica, Perú.

Teléfono: +51956747941

Email: Jorgeybaseta@yahoo.es

\section{Contribuciones De Autoría:}

SOWE, MPF, OTO, RCA:

Contribuciones en la concepción y

diseño del manuscrito, recolec-

ción, análisis e interpretación de

los datos; redacción y revisión

crítica del contenido del

manuscrito y la aprobación final del artículo.

Conflicto De Intereses: No

declarados.

Financiamiento: Autofinanciado.

\section{Como Citar}

Sauñe-Oscco Wilson E, Martínez Paucar Félix, Oscco-Torres Olinda, Ramos-Cajo Astrid. Nivel de ansiedad y proceso de afrontamiento en adultos mayores atendidos en un centro de salud ica perú 2017. Rev méd panacea 2019;8(1): 21-25

Recibido: 03 - 03 - 2019

Aceptado: 20 - 04 - 2019

Publicado: 23 - 04 - 2019

\section{RESUMEN}

Objetivo: Determinar el nivel de ansiedad y proceso de afrontamiento en los adultos mayores atendidos en un Centro de Salud Ica Perú 2017. Materiales y métodos: El estudio fue cuantitativo, de tipo descriptivo, y transversal, la muestra estuvo conformada por 62 adultos mayores que fueron seleccionados a través del muestreo no probabilístico intencional, la técnica que se utilizó fue la encuesta y entrevista. Resultados: En relación a los datos generales, el $56 \%$ es de sexo femenino, el $58 \%$ tienen entre 60 a 70 años, el $45 \%$ son empleados, el $89 \%$ presentan alguna enfermedad crónica, y el $45 \%$ vive con su cónyuge. Respecto al Nivel de ansiedad de los adultos mayores según escala de Hamilton el $58 \%$ presenta intensidad leve, 35\% no presenta ansiedad, 5\% intensidad media y el $2 \%$ tiene intensidad elevada. El Proceso de afrontamiento es desfavorable en la dimensión resolución de problemas, expresión emocional, apoyo social y evitación de problemas con $58 \%$, $61 \%$; $52 \%$, $53 \%$ y favorable en $42 \%$; $39 \%$; $48 \%$ y $47 \%$ obteniéndose un consolidado global desfavorable en $56 \%(35)$ y favorable en $44 \%(27)$. Conclusiones: El nivel de ansiedad es leve en la mayoría de los adultos mayores, aunque existe un $5 \%$ con intensidad media y $2 \%$ tiene intensidad elevada; el proceso de afrontamiento es desfavorable, por lo que se recomienda la valoración geriátrica integral así como sitios de encuentro entre pares con el fin que pueda socializar con más personas de su misma edad lo cual contribuirá en una mejor calidad de vida.

Palabras clave: Trastornos de ansiedad, Adaptación. ancianos (Fuente: DeCS BIREME)

\section{ABSTRACT}

Objective: To determine the level of anxiety and coping process in the elderly served in an Ica Peru 2017 Health Center. Materials and methods: The study was quantitative, descriptive, and cross-sectional; the sample consisted of 62 older adults who were selected through intentional non-probabilistic sampling, the technique used was the survey and interview. Results: In relation to the general data, $56 \%$ are female, $58 \%$ are between 60 and 70 years old, $45 \%$ are employed, $89 \%$ have some illness, and $45 \%$ live with their spouse. Regarding the anxiety level of the elderly according to the Hamilton scale, $58 \%$ present mild intensity, $35 \%$ have no anxiety, $5 \%$ average intensity and $2 \%$ have high intensity. The process of coping is unfavorable in the dimension of problem solving, emotional expression, social support and avoidance of problems with $58 \%, 61 \%$; $52 \%, 53 \%$ and favorable in $42 \% ; 39 \% ; 48 \%$ and $47 \%$ obtaining a global consolidated is unfavorable in $56 \%$ and favorable in $44 \%$ according to results. Conclusions: The level of anxiety is mild in the majority of older adults, although there is $5 \%$ with medium intensity and $2 \%$ with high intensity; the process of coping is unfavorable, which is why comprehensive geriatric assessment is recommended as well as meeting places between peers so that you can socialize with more people of the same age, which will contribute to a better quality of life.

Keywords: Anxiety disorders, Adaptation. elderly people (Source: DeCS BIREME) 


\section{INTRODUCCIÓN}

Según reportes de la OMS en el año 2017, la tendencia a sufrir desórdenes mentales va en aumento, sobre todo en los países de bajos y medianos ingresos, dado que la población en general está aumentando, pero según los últimos informes la esperanza de vida es menor en estos países, si bien es cierto en el siguiente decenio la población adulta mayor será mayor que los niños menores de 5 años, estos no tienen una esperanza de vida muy alta, llegando incluso a establecerse como tope los 80 años. Las cifras son alarmantes: al menos 322 millones de personas en el mundo sufren depresión, un $18 \%$ más que hace una década; y otros 264 millones padecen trastornos de ansiedad, lo que significa un incremento del $15 \%$ respecto a hace diez años. (1)

En la región de las Américas se estima que el 7,7 \% de las mujeres padece ansiedad, mientras que en el caso de los hombres es un 3,6 \%. Brasil es de nuevo el país de Latinoamérica que más desórdenes de ansiedad padece (9,3\% de la población); seguido de Paraguay $(7,6 \%) ;$ Chile (6,5 \%); Uruguay (6,4\%); Argentina (6,3\%); Cuba $(6,1 \%)$ y Colombia (5,8 \%). Les siguen Perú y República Dominicana (5,7 \%); Ecuador (5,6 \%); Bolivia (5,4\%); Costa Rica y El Salvador (4,6 \%); Nicaragua y Panamá (4,5 $\%)$; Venezuela (4,4\%); Honduras (4,3\%); Guatemala (4,2 $\%)$ y México (3,6\%). (2)

En el Perú según el Instituto Nacional de Estadística e Informática (INEI) en el año 2013, los adultos mayores representaban el $11.4 \%$ de la población total. Los mayores porcentajes se presentan en los departamentos de: Arequipa (7,3\%), Lima (7,2\%), Moquegua $(7,1 \%)$, Ancash y la Provincia Constitucional del Callao (7,0\% en cada caso) e Ica y Lambayeque (6,8\% en cada caso). Asimismo, las personas octogenarias superan la cifra de 334 mil y la mayoría son mujeres $(58,4 \%)$. (3)

Aproximadamente el $5 \%$ de la población adulta mayor es dependiente, es decir que hoy en día hay aproximadamente 138,588 personas adultas mayores que están limitados o impedidas para realizar sus actividades de la vida diaria y que les causa ansiedad, en consecuencia, requieren del apoyo de terceras personas. (4)

Los adultos mayores están expuestos a factores biológicos, psicológicos y sociales que afectan su salud mental. Los problemas más frecuentes son trastornos afectivos: por falta de apoyo familiar, fallecimiento de seres queridos o situaciones como la jubilación, y desórdenes del sueño que alteran significativamente la calidad de vida de los adultos mayores, entre otros. (5)

En Ica, en el año 2016, los resultados de la investigación realizada por Matta D., el 78\% (39) adultos mayores tienen nivel de ansiedad medio, el $14 \%$ (7) nivel de ansiedad alto y el $8 \%$ (4) nivel de ansiedad bajo. (6)
En la vida cotidiana, existen cambios naturales en los estados de ánimo, los problemas diarios que pueden producirse por dificultades familiares, económicas o laborales, son sorteados por las personas de acuerdo a sus capacidades individuales para enfrentar los conflictos. En ocasiones una persona puede experimentar estados pasajeros de tristeza cuando sus expectativas no son satisfechas o cuando enfrentan dilemas o contrariedades: decepciones amorosas, separaciones familiares, desempleo, desajustes conyugales, u otros.

En tal sentido el objetivo de la investigación fue determinar el nivel de ansiedad y proceso de afrontamiento en adultos mayores atendidos en un Centro de Salud de Ica 2017.

\section{MATERIALES Y MÉTODOS}

Se realizó una investigación cuantitativa, descriptiva y de corte transversal, haciendo uso del muestreo no probabilístico intencional, considerándose como referencia a 62 adultos mayores que se atienden en el Centro de Salud San José de los Molinos de Ica, se utilizaron instrumentos validados y confiables como la Escala de ansiedad de Hamilton el cual consta de 14 ítems con alternativas de respuestas tipo Likert, para el afrontamiento se utilizó el Inventario de estrategias de afrontamiento (CSI) validado por Tobin, Holroyd, Reynolds y Kigal, 1989 y adaptado por Cano, Rodríguez y García, 2006); consta de 20 ítems en 4 dimensiones, con alternativas de respuestas tipo Likert. El análisis estadístico de los datos se realizó haciendo uso del paquete informático Microsoft Excel y Microsoft Office Word 2007.

\section{RESULTADOS}

Según datos generales se obtuvo que el 56\%(35/62) es de sexo femenino, el 58\%(36/62) tienen entre 60 a 70 años, el $45 \%(28 / 62)$ son empleados, el $89 \%(55 / 62)$ presentan alguna enfermedad crónica, y el 45\%(28/62) vive con su cónyuge.

Respecto al Nivel de ansiedad de los adultos mayores según escala de Hamilton el 58\%(36/62) presenta intensidad leve, 35\%(22/62) no presenta ansiedad, $5 \%(3 / 62)$ intensidad media y el $2 \%(1 / 62)$ tiene intensidad elevada.

Según el Proceso de afrontamiento es desfavorable en la dimensión resolución de problemas, expresión emocional, apoyo social y evitación de problemas con $58 \%(36 / 62), \quad 61 \%(38 / 62) ; \quad 52 \%(32 / 62), \quad 53 \%(33 / 62)$ y favorable en $42 \%(26 / 62) ; 39 \%(24 / 62) ; 48 \%(30 / 62)$ y $47 \%(29 / 62)$ obteniéndose un consolidado global es desfavorable en $56 \%(35 / 62)$ y favorable en $44 \%(27 / 62)$ según resultados. 
Tabla 1: Datos generales de los adultos mayores atendidos en el Centro de Salud de San José de los Molinos, Ica Perú 2017.

\begin{tabular}{|c|c|c|}
\hline DATOS GENERALES & $\mathbf{F r}$ & $\%$ \\
\hline \multicolumn{3}{|l|}{ SEXO } \\
\hline Masculino & 27 & 44 \\
\hline Femenino & 35 & 56 \\
\hline \multicolumn{3}{|l|}{ EDAD } \\
\hline 60 a 70 años & 36 & 58 \\
\hline 71-80 años & 19 & 31 \\
\hline 81 a más años & 7 & 11 \\
\hline \multicolumn{3}{|l|}{ OCUPACIÓN } \\
\hline Empleado & 28 & 45 \\
\hline Eventual & 20 & 32 \\
\hline No trabaja & 14 & 23 \\
\hline \multicolumn{3}{|c|}{ PRESENTA ALGUNA ENFERMEDAD CRÓNICA } \\
\hline $\mathrm{Si}$ & 55 & 89 \\
\hline No & 7 & 11 \\
\hline \multicolumn{3}{|l|}{ CON QUIEN VIVE } \\
\hline Con su cónyuge & 28 & 45 \\
\hline Con su cónyuge e hijos & 18 & 29 \\
\hline Con otros familiares & 6 & 10 \\
\hline Vive solo(a) & 10 & 16 \\
\hline TOTAL & 62 & 100 \\
\hline
\end{tabular}

Gráfico 1: Nivel de ansiedad según Escala de Hamilton de los adultos mayores atendidos en el Centro de Salud de San José de los Molinos, Ica Perú 2017

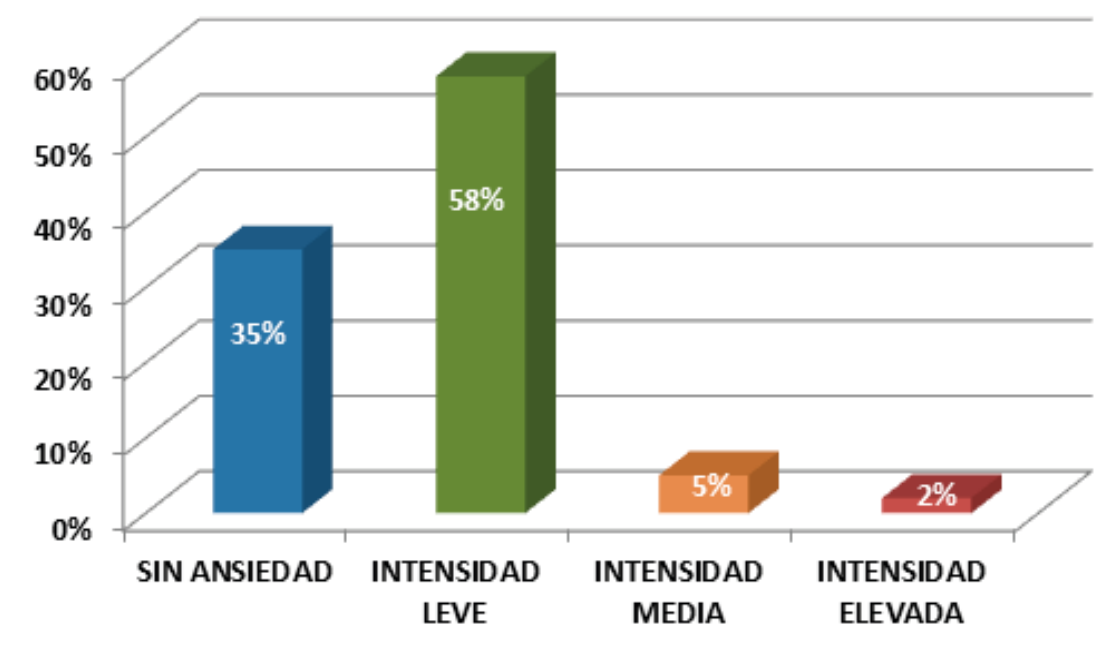


Gráfico 2: Proceso de afrontamiento global de los adultos mayores atendidos en el centro de salud de San José de los Molinos, Ica Perú 2017.

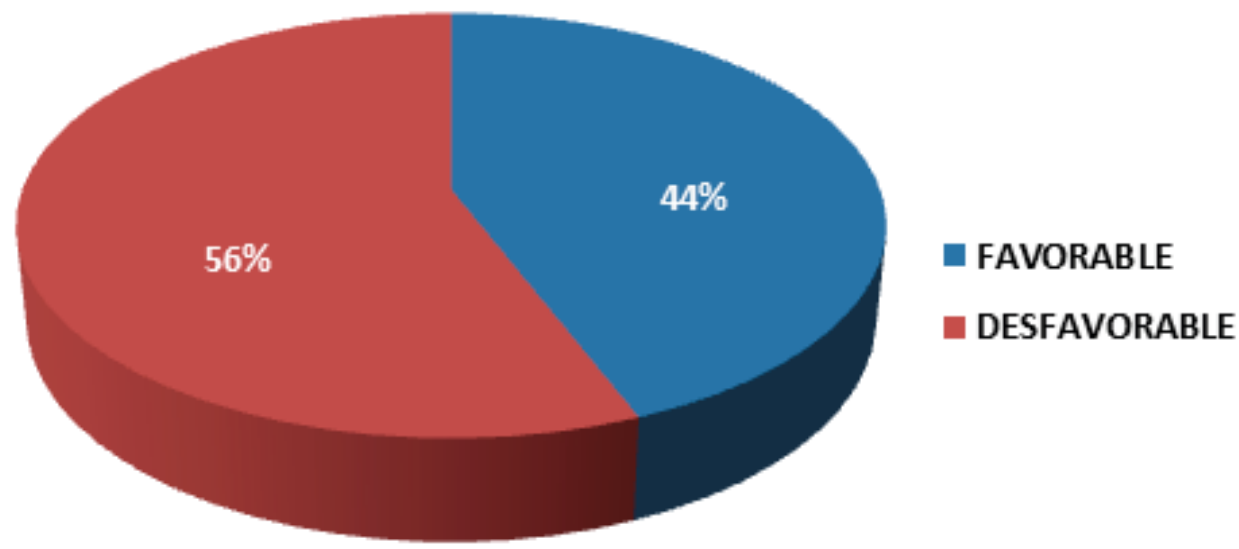

\section{DISCUSIÓN}

Referente a los datos generales de los adultos mayores en estudio, los resultados son similares con Calcina Y. (7) tienen entre 60 y 74 años $55.8 \%$ (39), según el sexo femenino $75 \%$ (39), estado civil son casados(as) $59.6 \%$ (31).

El proceso de envejecimiento varía de un individuo a otro por lo cual no se deben esperar las mismas características en cierta edad puesto que no todos los sistemas funcionan de la misma forma. Se observa un porcentaje que son empleados y viven con su cónyuge lo cual es una fortaleza el hecho de contar con una pareja que les brinde ayuda y apoyo emocional para resolver problemas y enfrentarse a las adversidades que se les presenta en su estado de salud. Por lo presentado los familiares deben estar preparados para brindar el cuidado que requieren los adultos mayores debido a que existe un crecimiento demográfico en los adultos mayores y no se está tomando las precauciones para cubrir la demanda de salud que se requiere.

Respecto al nivel de ansiedad, los resultados son contrarios con Mamani D. (8) El nivel de ansiedad de mayor porcentaje fue la ansiedad severa $72.7 \%$ (8) en San Salvador de Puno y 68.8\% (11) en Virgen del Rosario de Chucuito, y un menor porcentaje con ansiedad moderada 27.3\% (3) en San Salvador de Puno y 31.3\% (5) en Virgen del Rosario de Chucuito, Nuestros resultados son similares con los hallados por Buitrago L, Cordón L. (9) donde los niveles de ansiedad presentados en los adultos mayores son bajos $66.7 \%$ (10) marcaron si y el
$33.3 \%$ marcaron no.

Respecto a los resultados se puede inferir que se producen cambios vitales en la vejez, debido a ello sentir ansiedad en algún momento no debe ser sinónimo de alarma pues se trata de un tipo de reacción habitual ante situaciones difíciles como consecuencia de la vejez con la función de activar al organismo ante situaciones que considera de riesgo.

Referente al proceso de afrontamiento, nuestros resultados son similares con Calcina Y. (7) El nivel de afrontamiento-adaptación de los adultos mayores se encuentra en un bajo nivel, en un $63.5 \%$ teniendo a los factores: "conocer y relacionar" en un porcentaje menor (47) $90.4 \%$, seguido del factor "procesamiento sistemático" (46) 88.5\%. Colque E, Velásquez V. 13 los resultados fueron que el afrontamiento - adaptación es bajo en $44 \%$, de los adultos mayores.

En general, las habilidades de afrontamiento son importantes tanto en la prevención como en la adaptación debido a que el envejecimiento disminuye la capacidad física constituyéndose así en una fuente principal de estrés físico y emocional; y no es fácil aceptar que no pueden controlar aspectos básicos de su vida y también que van perdiendo autonomía, debiéndose en estos casos promocionar un envejecimiento digno, activo, solidario y saludable haciendo énfasis en los cuidados preventivos, la autoayuda así como la autonomía de los adultos mayores. 


\section{REFERENCIAS BIBLIOGRÁFICAS}

1. OMS. Envejecimiento [Datos de internet]. [sitio web]. Fecha de acceso 5 de julio de 2017. Disponible en: http://www.who.int/topics/ageing/es/

2. Melía H. Depresión y ansiedad, dos males que golpean cada vez más fuerte. Informe OMS. URL disponible

en:

https://www.eldia.com/nota/2017-3-5-4-36-9-depr esion-y-ansiedad-dos-males-que-golpean-cada-ve z-mas-fuerte de Google.

3. INEI. Día mundial de la población. 2013. Disponible e $n$

http://www.unfpa.org.pe/Articulos/Articulos/INEI-E stado PoblacionPeruana-2013.pdf.

4. Ortega Y. Depresión y ansiedad aumentan un 30\% el riesgo de eventos cardiovasculares en personas con síndrome metabólico. [Datos de internet]. 2017 Área geriátrica.

5. Ministerio de Salud. ¿Por qué es importante cuidar la salud mental de los adultos mayores? Sala de prensa. Febrero 2018 URL disponible en: http://www.rpmesp.ins.gob.pe/index.php/rpmesp/ article/view/2211/2165
6. Matta D, Ansiedad y percepción de abandono en adultos mayores del servicio de cirugía del hospital regional. Ica, [Tesis licenciatura]. 2016. Disponible en: http://repositorio.upsjb.edu.pe/handle/upsjb/1283

7. Calcina Y. Capacidad funcional y afrontamiento adaptación en adultos mayores del Club "Años Dorados" Municipalidad de Hunter, Arequipa - 2014. Disponible en: http://repositorio.unsa.edu.pe/bitstream/handle/UN SA/2265/ENcaquyp.pdf?sequence $=1$

8. Mamani D. Depresión, ansiedad y estado nutricional del adulto mayor albergado en los centros de atención residencial de la provincia de Puno y Chucuito. 2017. URL: Disponible en: http://repositorio.unap.edu.pe/handle/UNAP/5056F echa: 2017-08-02

9. Buitrago L, Cordón L. Niveles de ansiedad y estrés en adultos mayores en condición de abandono familiar de una institución geriátrica del municipio de Facatativá. Colombia. 2017. Disponible en: http://dspace.ucundinamarca.edu.co:8080/xmlui/bit stream/handle/123456789/561/Trabajo\%20de\%20g rado.pdf? sequence $=2$ \&isAllowed $=y$ 